\title{
OPTIMISING THE REFERENCE POINT WITHIN A JOURNAL BEARING USING LASER ALIGNMENT
}

\author{
Ty Aaron Smith ${ }^{1)}$, Guixin Fan') \\ Natalia Nikolova ${ }^{1,2)}$, Kiril Tenekedjiev ${ }^{1,2)}$ \\ 1)Australian Maritime College, University of Tasmania (Australia) \\ ${ }^{2)}$ Nikola Vaptsarov Naval Academy (Bulgaria)
}

\begin{abstract}
This paper observes different alignment conditions in a journalbearing-supported machine (i.e. propeller bearings within the stern tube of a marine vessel). A test rig is built so that the driven machine has fixed supports, roller bearings in an electrical motor. An alignment tolerance is met at the coupling of the journal, which is positioned within three journal bearings. This research can be applied to any industry with machines of the same characteristic. The journal bearings are designed to simulate the large tolerance seen in a vessel's stern tube only for lubrication control oil is used for the bush bearings. Various sensors are placed on the test rig to record data for later machine analysis. Using proximity probes and accelerometers the vibration of the journals and ball bearings can be visualised using orbital plotting and Fast Fourier Transform (FFT) through both LabVIEW and MATLAB respectively. Four alignment procedures are applied to the coupling using a laser unit. The first two will be so that the offset and angular misalignment is produced in the FFT spectrums as per ISO10816 Mechanical vibration so that a reference point is formed for the test rig. The other two alignment procedures represent current industry practice. These procedures are then compared to see which is the preferred reference point that will optimise the reliability value of a whole system. Finding the procedure with minimal vibration will increase the design life of housing structures for such machines, which will increase the overall profit of the shipping industry. Our developments and procedures will be further utilized in practical classes to improve maritime engineering education.

Keywords: Preventive maintenance; LabVIEW; MATLAB test rig; proximity probes; accelerometers; flowrate
\end{abstract}

\section{Introduction}

Optimising the reference point within a journal bearing is an oversight seen in engineering industry. Two factors stick out, one being the alignment of a propulsion system within a vessel and the second discrepancy is the technician 
not understanding how to follow the graphical user interface of a laser unit used for shaft alignment (Piotrowski 2006).

In this paper, we shall explore different alignment conditions in a journalbearing-supported machine (namely, the propeller bearings within the stern tube of a marine vessel). We shall describe the test rig created to provide fixed supports and roller bearings in an electrical motor of the machine. We shall apply four alignment procedures to the coupling using a laser unit. These procedures are then compared to see which is the preferred reference point that will optimise the reliability value of a whole system. Finding the procedure with minimal vibration will increase the design life of housing structures for such machines, which will increase the overall profit in shipping industry. The procedures can also be applied for training purposes in maritime engineering.

In what follows, section 2 presents the technical background and preparation for our analysis. Section 3 presents the structure of the test rig and the four alignment procedures that we shall test. Section 4 concludes the paper.

\section{Technical background}

Shaft alignment and laser alignment equipment

Shaft alignment is when two shafts are required to operate as close to colinear as possible. As carrying out alignment procedures is time-consuming in the industry, a tolerance is given, which allows for a minimal load to be applied to the bearing. The tolerance is applied to the coupling between two separate shafts. Tolerance is shown in the vertical and horizontal with offset, and angular parameters in Figure 1.

Laser alignment is equipment allowing to improve preventive maintenance which increases the reliability of the systems. The benefit of using this equipment allows the user to get fast results with high accuracy due to their graphical user interface (GUI), as it can be quickly followed (see Figure 2). As the procedure of alignments gets easier for personnel using the GUI laser units, the most important part is being neglected during complicated alignments, such as those on ships. The reference point of an alignment job varies since each alignment is different.
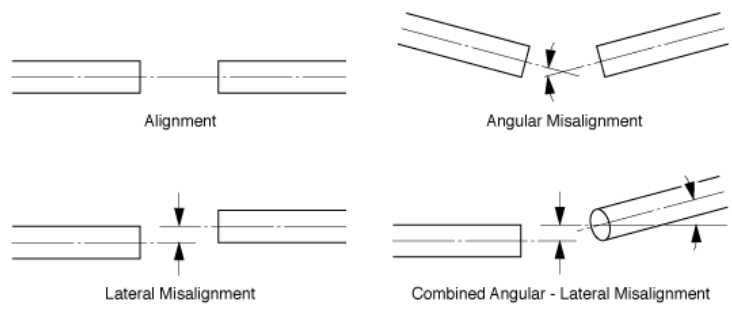

Figure 1 - Common Shaft Misalignments

Figure 1. Results of shaft alignment (SDP/SI 2020) 


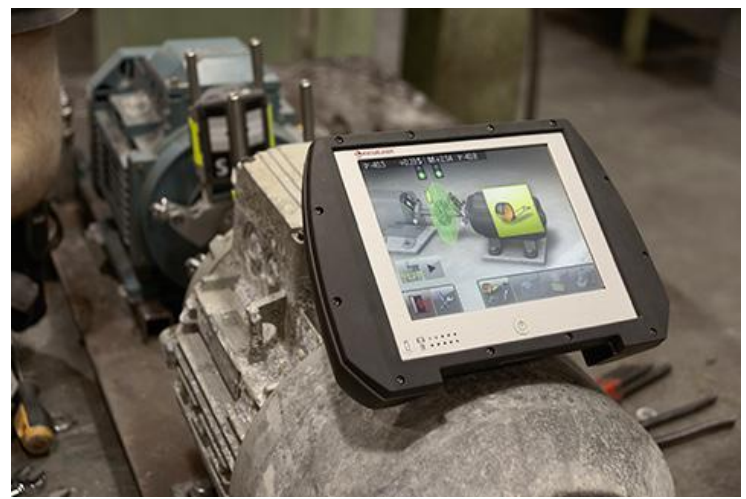

Figure 2. Laser shaft laser alignment (ACOEM Fixturelaser 2020)

\section{Vibration Analysis}

Vibration analysis is one of the most potent diagnostic practices that allows for diagnosis of faults from patterns that dictates the error to the rotating machinery while it is running.

An accelerometer, given in Figure 3., that has a piezoelectric material (Lisson 2017) that reacts to the force of the vibration, measures the acceleration of motion that is applied to a structure ${ }^{1)}$.

The Fast Fourier Transform (FFT) takes a time waveform and transforms the vibration signal into a frequency domain. The practice is widely used as it is exceptionally powerful for diagnosing rotating machinery. Figure 4. shows a 3D transformation of the vibration signal being transferred from using the formula illustrated.

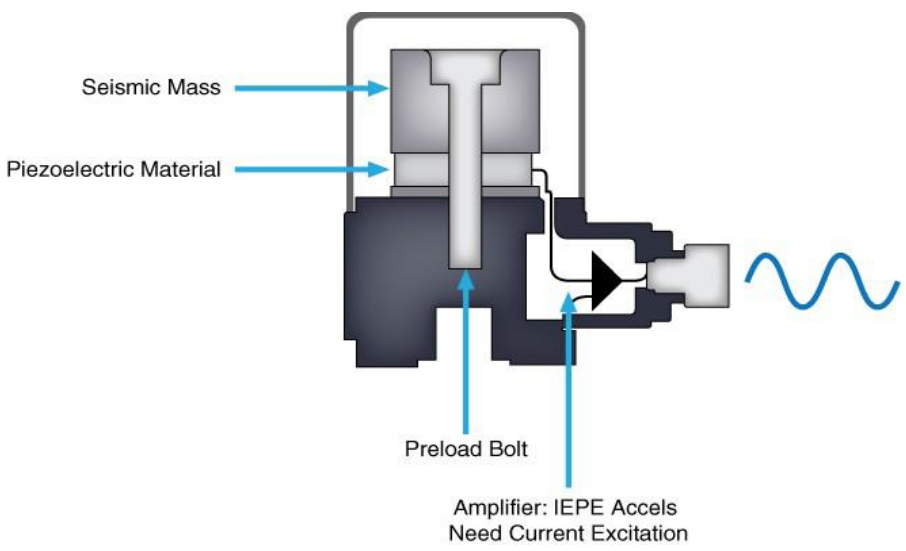

Figure 3. Accelerometer Labelled Diagram (National Instruments 2020) 


\section{Fourier Transform - Review}

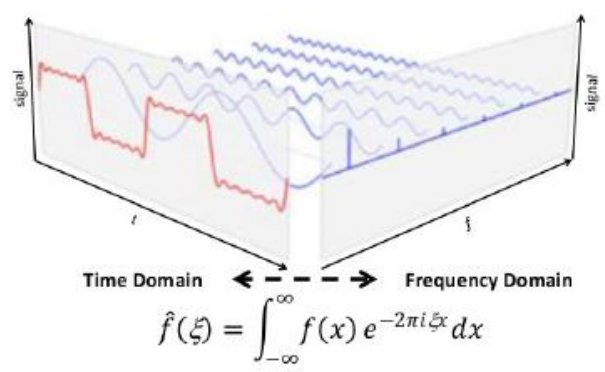

Figure 4. Demonstration of Fourier transform (Weixiang 2016)

Misalignment occurs when the centre lines of the coupled shafts are not collinear. Such phenomenon produces frequency peaks. The spectrum will show two different types of faults - parallel and angular misalignment, as seen in Figure 5.

An Eddy current sensor (Figure 6) is used by producing a flux field between the sensor and the journal. During the running condition of the journal, the movement of the journal is achieved in a single axis. During the displacement of the journal, the flux produces different singles that becomes recordable data for aliasing.

Orbital plotting gives a lot of information on how the journal is performing. If set up correctly, the eddy current sensors will provide a visualisation of where the journal is positioned in the bearing, as shown on Figure 7.

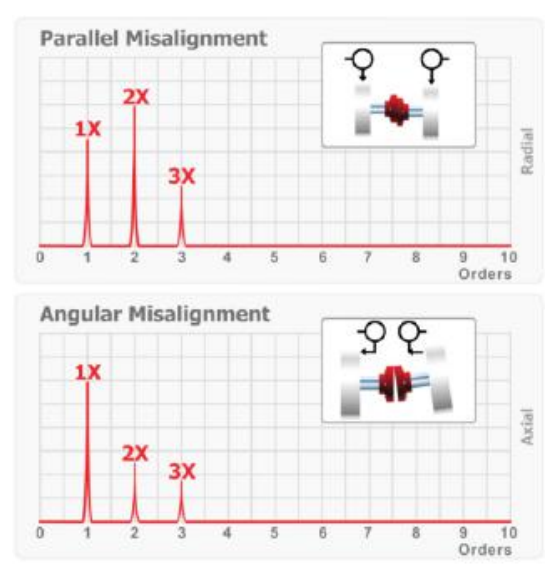

Figure 5. Parallel and angular misalignment (Mobius Intitute 2020) 


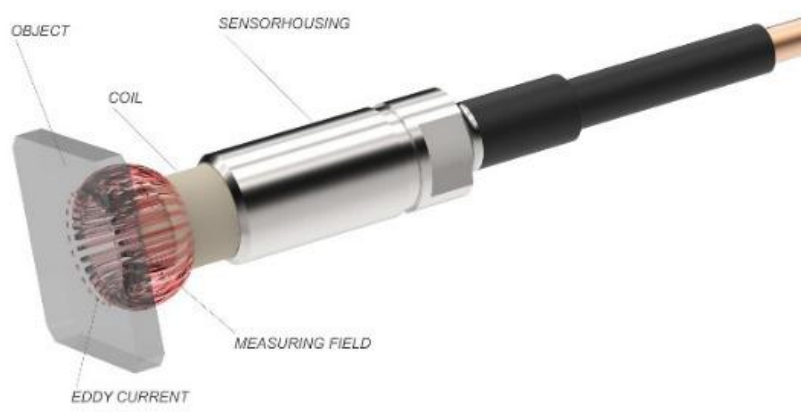

Figure 6. Eddy current sensor (eddylab 2020)

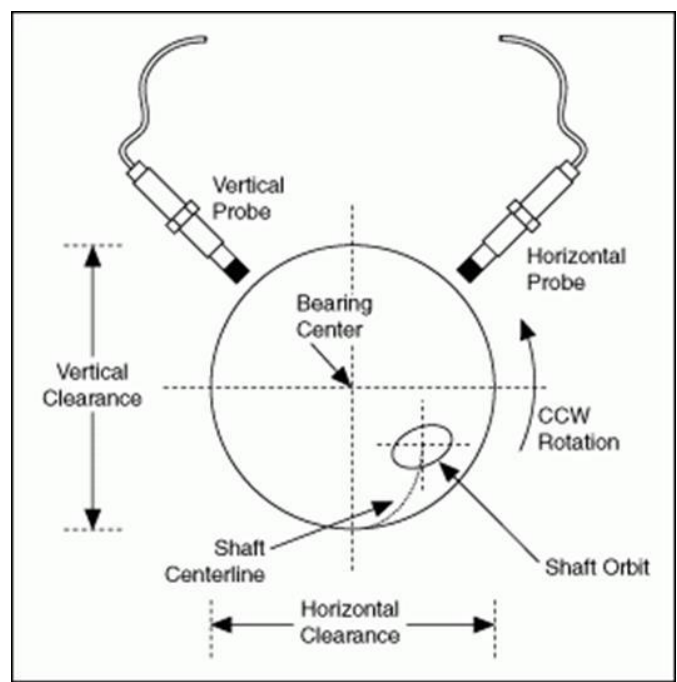

Figure 7. Proximity probe sensor setup (Slinger 2017)

Displacement probes are positioned 90 degrees apart from each other on bearing housing. The probes, and the Eddy current sensors, create a signal from the movement of the journal in one axis. The displacement measurement of the reference point is then plotted onto a cartesian plane, forming the orbital plot. The shape of the orbit is then analysed to detect the status of the journal, - normal, unbalanced or shaft misalignment as shown in Figure 8. 


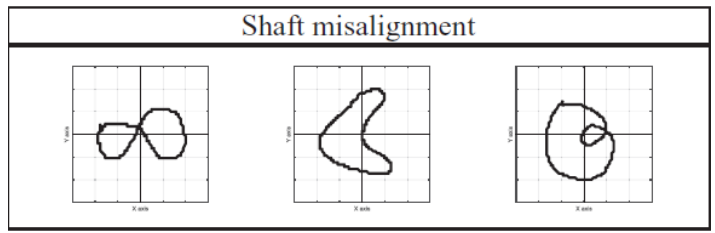

Figure 8. Different orbit shapes according to fault types

\section{Journal Lubrication Flow}

Using the parameters from the journal bearing, the journal oil flow can be calculated, ensuring that the correct amount of lubrication is applied to a journal bearing. Figure 9 and 10 show a labelled diagram of a journal bearing that aids the oil flow Gallons Per Minute (GPM) Equation.

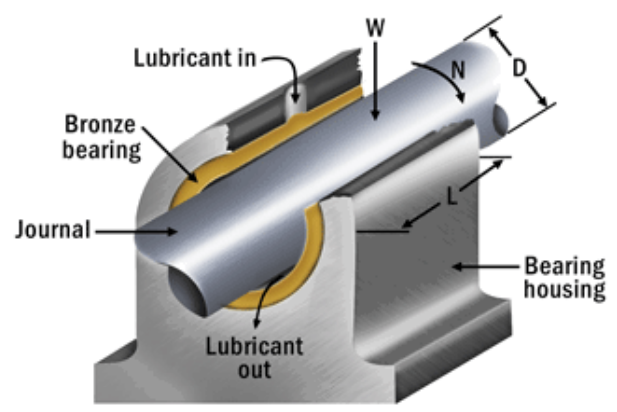

Figure 9. Typical journal bearing (Noria Corporation 2020)

The Gallons Per Minute (GPM) equation is given as (Noria Corporation 2020):

$$
\begin{aligned}
& Q=29.3 \times 10^{-9}\left(L+.0043\left(\frac{W}{D}\right)\right) m D^{2} N \\
& m=1000\left(2 \frac{C}{D}\right)
\end{aligned}
$$

where D-journal bearing (in inches), L-bearing length (in inches), m-bearing clearance factor, N-rotational speed, W-load to be supported (in pounds).

\section{Structure of the test rig}

The test rig (in its finite element analysis (FEA), Computer Aided Design (CAD) and manufacturing structure given in Figures 11, 12, 13) is designed to have similar characteristics of a stern tube in a ship. A stern tube typically houses two bearings and a steady bracket, aka 'A' bracket (or 'P' bracket), which houses a 
single bearing. Therefore, to simulate such configuration, three bearings are placed at the start of the test rig.

The prime mover of the drive train on this rig is an electrical motor which will include the drive unit with thrust, i.e., the fixed bearing of the drive train. Such bearing sets the angle and offset vector through the other bearings when aligned at the coupling. Note that the results obtained from this setup can be applied to any other machine with journal bearing configurations.

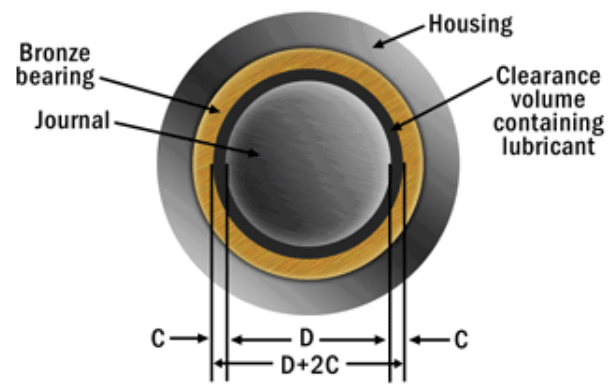

Figure 10. Typical journal bearing 2 (Noria Coporation 2020)

Different alignment conditions will be simulated at the coupling between the driven shaft and the electric motor (Driveshaft). Each journal bearing has two Eddy current sensors, and one additional sensor is placed at each sump of the bearings to record the temperature, as each bearing will have a different state of misalignment, and the friction from close contact in the bearing will cause rise in temperature. Each bearing has its own lubrication pump, actuated by a variable speed drive (VSD), which self-controls the oil flow through a flow gauge. A pressure sensor is placed after the flow gauge to observe any change of oil pressure when misalignment occurs. As oil heats up due to friction from the contact between oil pump and bearing, an oil cooler has been installed to ensure constant temperature that suits the viscosity of the oil, thus ensuring proper bearing lubrication.

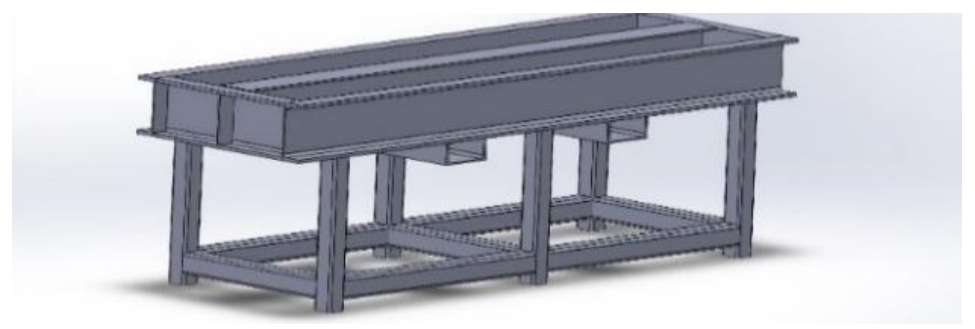

Figure 11. Bearing CAD and FEA design 


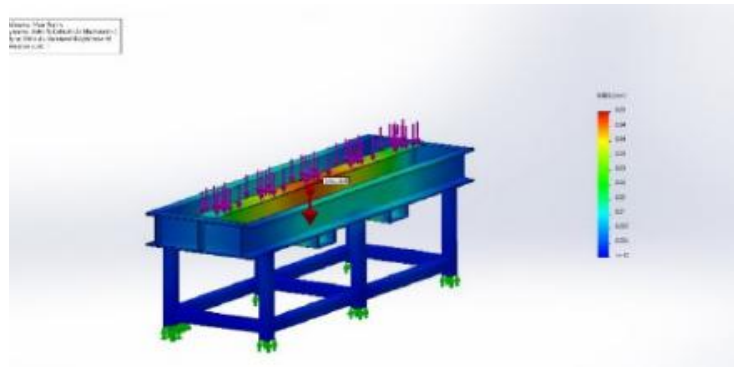

Figure 12. FEA on frame of the test rig

To maintain constant oil temperature delivered to each oil pump, the pickups for the pumps are at the same point on the opposite ends of the tank where the return from the bearing sump is located. At the pickup of the oil tank, a temperature sensor is positioned to ensure the temperature reaches equilibrium throughout each run of the experiment.

To clarify that misalignment is present, two tri-axel accelerometers are placed on the electric motor at the drive end (DE) and the non-drive end (NDE). Signal from the accelerometers is retrieved as time waveform that will be converted by FFT within a spectrum so the patterns can be analysed for misalignment. Figure 14 shows the variable speed drivers (VSD) being wired and the configuration of the data acquisition (DAQ) card. The test rig in its final stage, ready for testing, with a TV stand for visual aid, shown in Figure 15.

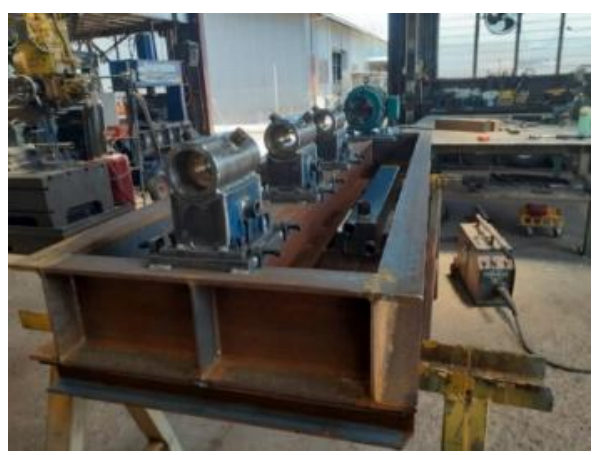

Figure 13. Test rig being manufactured in workshop

\section{First Procedure}

Parallel misalignment is simulated in the test rig and the journal bearing pattern is observed (strong two times peak in an FFT with one and three times present) for later comparison with the third and fourth procedures. Raw data from the motor triaxles is entered into an FFT function in both MATLAB and LABVIEW producing 
a graph for visual analysis. Using Laser alignment technology, the motor is aligned to the journal ensuring an offset alignment is achieved to produce the frequencies needed to set a reference point for the test rig.

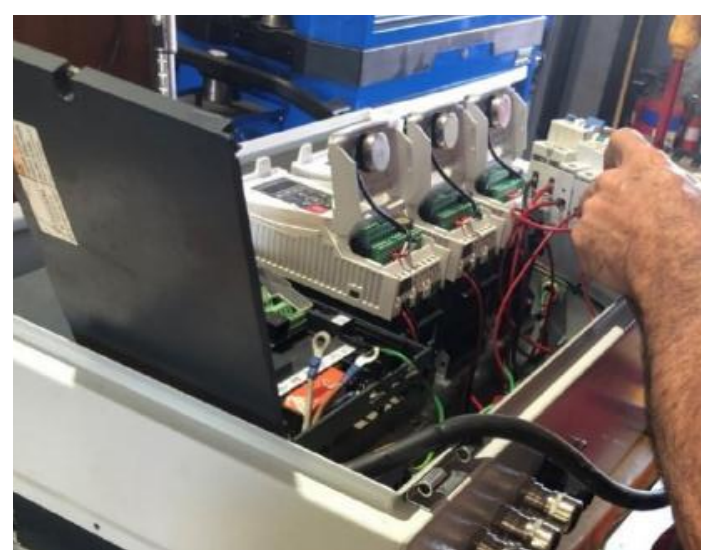

Figure 14. Wiring the variable speed drivers

\section{Second Procedure}

Angler Misalignment is simulated in the test rig and the journal bearing pattern is observed (strong one peak with a steady two-times and three-times peak within the FFT) for later comparison with the third and four procedures. Raw data from the motor triaxles is entered into an FFT function in both MATLAB and LabVIEW producing a graph for visual analysis. Using Laser alignment technology, the motor is aligned to the journal ensuring an offset alignment is achieved to produce the frequencies needed to set a reference point for the test rig.

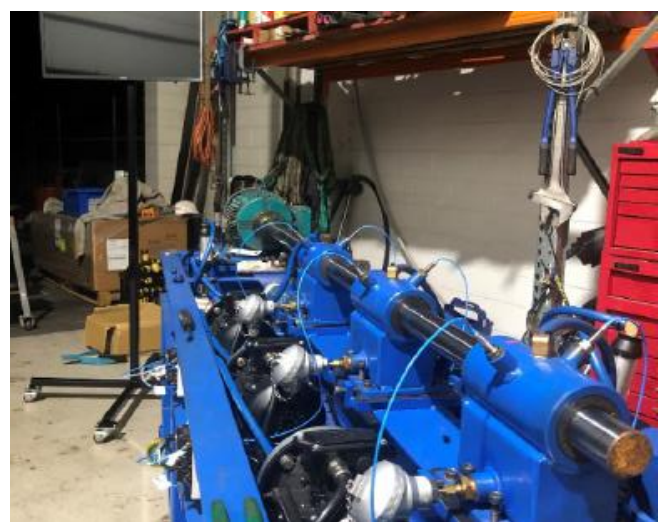

Figure 15. Structure of the test rig 


\section{Third Procedure}

The third procedure involves using one of two industry reference points when aligning a fixed bearing to a journal bearing. A bump test is carried: the journal bearing is lifted off the bottom of the bearing to reach the top of the bearing. With this movement, a dial in place measures the displacements in the vertical axis. Dividing this measurement by two, the midpoint of the first bearing can be set. The motor is aligned to suit the journal reference point and the test rig ran up to record the data.

\section{Fourth Procedure}

The fourth procedure uses the second common method from industry. The reference point is used when aligning a fixed bearing to a journal bearing. To eliminate shaft sage, the journal is left on the bottom of the bearing. Using laser technology, an offset is applied to the laser unit setting. A bump test is conducted to measure the vertical movement within the journal bearing, captured using a dial indicator. A dial in place measures the displacements in the vertical axis. Dividing this measurement by two the midpoint of the first bearing can be set. The motor is aligned to suit the journal reference point and the test rig ran up to record the data.

\section{Conclusions}

Our constructed test rig meets high expectations with visualisation of the movements within a ball bearing and a journal bearing. The realistic values simulated on the FEA of the test rig was rewarding ensuring no further modifications needed for operations under dynamic conditions of the test rig. The DAQ card configuration was optimised with the level of skill that the author was taught; other configurations could have been employed to lower the project budget. The preferred alignment procedure is the fourth procedure, showing minimal vibration, i.e., minimal damage to parts, thus increasing the overall reliability of the system.

As future steps in this research, the test rig data recorded using the DAQ card will be iterated through both LabVIEW and MATLAB. This will produce graphs in the frequency domain and orbital plotting. The outcome from all four alignment procedures will be subjected to further analysis to identify optimum procedures and settings. Another area of future development is the implementation of the procedures and the test rig functionality in practical training of engineering students thus contributing to improvements in maritime education and training.

Acknowledgments. The authors would like to express their gratitude to Preston Engineering for their input for the design of the test rig. Adapting such a 
design with available machineries can only be done with experienced tradesman, fitter and turners.

The authors also would like to thank Fluid Power Engineering for ensuring that we achieve the correct outcome with the most economical approach to the task in regards to the lubrication systems.

The authors would like to thank Castle Engineering for their guidance and input, which helped the wiring of the test rig with both alternating current $(\mathrm{AC})$ and direct current (DC) that needed an 'A' class electrician to wire up and sign off to meet Australian safety. They ensured that the DAQ card meets the parameters that the National Instruments required to minimize potential interference with signal transfer through cabling.

The authors express their gratitude to the NMIS team for their constant support, space and input ensuring the project could be contacted. Warehouse space was given to carry out the experiment during COVID regulations.

The authors also wish to thank Dr Hung Hguyen for his guidance and advise on how to purchase the National Instrument from Stuart Little, Braemac.

\section{NOTES}

1. OMEGA A spectris company, 2020. Accelerometers. Available from: https:// au.omega.com/prodinfo/accelerometers.html

\section{REFERENCES}

ACOEM Fixturelaser, 2020. Shaft Alignment. Available from: https://www. fixturlaser.com/Shaft-Alignment/

Eddylab, 2020. Eddy Current Probes. Available from: https://www. eddycurrentprobe.com/

Heideman, M.T., Johnson, D.H. \& Burrus, Ch. S., 1984. Gauss and the history of the fast Fourier transform. IEEE ASSP Magazine. 1(4), 14 - 21.

Lisson, D.L., 2017. Materials Technology. $10^{\text {th }}$ edition. Australian Maritime College (UTAS).

National Instruments, 2020. Measuring Vibration with Accelerometers. Available from: https:/www.ni.com/en-au/innovations/white-papers/06/ measuring-vibration-with-accelerometers.html

Noria Corporation, 2020. Determining Proper Oil Flow to Journal Bearings . Available from: https://www.machinerylubrication.com/Read/29654/ journal-bearing-oil

Piotrowski, J., 2006. Shaft Alignment Handbook. $3^{\text {rd }}$ Edition. Parkway NW, CRC Press.

SDP/SI., 2020. Couplings. Available from: https://sdp-si.com/catalogs/ D757-Couplings-Universal-Joints.php 
Slinger, G., 2017. Fluid film bearings. Available from: https://www.pioneerengineering.com/resources/fluid-film-bearings

Weixiang, C., 2016. Algorithm: Fourier Transform. Available from: http:// chenweixiang.github.io/2016/06/14/fourier-transform.html

\author{
$\triangle$ Ty Aaron Smith \\ https://orcid.org/0000-0001-6803-7967 \\ Australian Maritime College \\ University of Tasmania \\ Launceston, TAS, Australia \\ E-mail: tasmith9@utas.edu.au,ty@nmis.com.au
}

$\triangle$ Guixin Fan

https://orcid.org/0000-0002-0334-2271

Australian Maritime College

University of Tasmania

Launceston, TAS, Australia

E-mail: gfan@utas.edu.au

$\triangle$ Natalia Nikolova

https://orcid.org/0000-0001-6160-6282

Australian Maritime College

University of Tasmania

Launceston, TAS, Australia

Nikola Vaptsarov Naval Academy

Varna, Bulgaria

E-mail: Natalia.Nikolova@utas.edu.au

$\triangle$ Kiril Tenekedjiev

https://orcid.org/0000-0003-3549-0671

Australian Maritime College

University of Tasmania

Launceston, TAS, Australia

Nikola Vaptsarov Naval Academy

Varna, Bulgaria

E-mail: Kiril.Tenekedjiev@utas.edu.au 